\title{
Peptide Sequence Determination by Matrix- Assisted Laser Desorption Ionization Employing a Tandem Double Focusing Magnetic-Orthogonal Acceleration Time-of-Flight Mass Spectrometer
}

\author{
Katalin F. Medzihradszky, Gregory W. Adams, and Alma L. Burlingame \\ Department of Pharmaceutical Chemistry, Mass Spectrometry Facility, University of California, San Francisco, \\ San Francisco, California, USA
}

Robert H. Bateman and Martin R. Green

VG Organic, Manchester, UK

\begin{abstract}
This report describes the fragmentation processes for peptides induced by collisional activation of the ${ }^{12} \mathrm{C}$ isobar of matrix-assisted laser desorption ionization (MALDI)-generated pseudomolecular ions employing an EBE orthogonal acceleration time-of-flight mass spectrometer and using xenon as the collision gas at a laboratory collision energy of $800 \mathrm{eV}$. These MALDI-collision-induced dissociation (CID) spectra are shown to provide sequence information of comparable quality to those obtained by using high energy CID conditions with liquid secondary ionization mass spectrometry on a four-sector tandem instrument. Peptide sequencing via MALDI-CID is demonstrated on three tryptic peptides obtained from a bacterial protein (P450 isozyme) of unknown sequence. Sensitivity is shown to be at the 1 pmol level for standard peptides. (J Am Soc Mass Spectrom 1996, 7, 1-10)
\end{abstract}

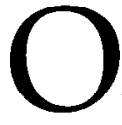
ver the past few years the discovery of soft ionization techniques has revolutionized the ways that problems in protein biochemistry may be tackled through the use of a variety of new mass spectrometric methods and instrumentation. Progress has been widespread and rapid, from the molecular weight mapping of protein digests to the amino acid sequence determination and the structural characterization of covalent modifications (for reviews, see [1] and [2]).

Because these soft ionization techniques tend to form ions by protonation-deprotonation of the neutral molecule with low vibrational energy content, signals due to the intact pseudomolecular ion(s) are observed, which provides a sensitive means for determination of the molecular weights and purity of substances or the composition of mixtures. In addition, some fragments are observed in the cases of liquid secondary ionization (LSI) and matrix-assisted laser desorption ionization (MALDI) as well, although the fragments are of unpredictable qualitative nature and relative abun-

Address reprint requests to Professor A. L. Burlingame, Department of Pharmaceutical Chemistry, University of California, San Francisco, 513 Parnassus, C18, San Francisco, CA 94143-0446. dance. To exploit fully a mass spectrometric strategy for structural elucidation of such gas-phase molecular species, enhancement of their vibrational energy is required to induce unimolecular dissociation to fragments that are characteristic of the structure of the molecule. This is accomplished readily, for example, by collision with an inert gas such as helium, followed by a second stage of mass analysis to record the fragment ions thus generated, which yields collisioninduced dissociation (CID) spectra. Two distinct collision regimens are in common use: so-called low energy and high energy; they impart different amounts of internal energy, which results in the induction of a variety of ion types, some of which are unique to the high energy process [3]. These high energy ion types have been shown to be of considerable value in the de novo determination of complete and unambiguous amino acid sequence $[4,5]$.

Because matrix-assisted laser desorption ionization (MALDI) is believed to provide the highest ionization efficiency for peptides, it is of major importance for protein characterization [6] and has been used for digest mapping and identification of proteins isolated from two-dimensional gels [7, 8]. Although mapping strategies have enjoyed some success and utility in 
connection with identification of known proteins in cell lysates through database searching [7], it is of considerable importance to be able to generate high quality reproducible CID spectra with the inherent information content for direct sequence and structural analysis. For this purpose, the development of a combination of the high ionization efficiency of MALDI with the high resolution and mass accuracy of double-focusing instruments should provide the high energy CID information sought. A tandem mass spectrometer that is compatible with pulsed ionization techniques and consists of a double-focusing instrument and a co-linear time-of-flight analyzer with a reflectron has been described by Russell's group [9, 10]. The same group reported a CID spectrum of the MALDI-generated molecular ion of angiotensin II (10 pmoles loaded) [11] acquired on this instrument [12]. This CID spectrum showed very unusual fragmentation, which suggested that the accuracy of mass measurement as well as the resolution would need improvement. In addition, by using MALDI, the CID spectrum of substance $P$ has been recorded with xenon as a collision gas on a four-sector instrument with array detection [13]. In a further preliminary report, the MIT group [14] has presented the photodissociation spectra of substance $P$ and renin substrate from MALDI experiments.

In this article we report the sequence analysis of peptides by using MALDI and a new ion optical strategy based on a tandem instrument comprised of an EBE geometry for MS-1 and an orthogonal acceleration time-of-flight analyzer for MS-2 [15]. This instrumentation provides the means to generate high energy CID spectra from MALDI pseudomolecular ions with monoisotopic precursor ion selection, and holds promise for the characterization of unknown proteins available in the femtomole range. In addition, both liquid secondary ion mass spectrometry (LSIMS) foursector high energy CID and MALDI postsource decay (PSD) mass spectra are presented on synthetic peptides for comparative evaluation.

\section{Experimental}

\section{Tryptic Peptides}

Approximately 100 pmol of a purified soluble unknown bacterial $\mathrm{P} 450$ protein were incubated with trypsin ( $4 \% \mathrm{w} / \mathrm{w}$; Worthington, Freehold, NY) in 50 $\mathrm{mM} \mathrm{NH} \mathrm{HCO}_{3}$ buffer $(\mathrm{pH} 7.8)$ at $37^{\circ} \mathrm{C}$ for $18 \mathrm{~h}$. The tryptic peptides were separated by reversed-phase high-performance liquid chromatography (HPLC) on a microbore $\mathrm{C} 18$ column $(250 \times 1 \mathrm{~mm}$; Vydac, Hesperia, CA) by using $0.1 \%$ trifluoroacetic acid (TFA) in water as solvent $\mathrm{A}$ and $0.08 \%$ TFA in acetonitrile as solvent $B$. The amount of solvent $B$ was increased by $1 \%$ per minute during the gradient elution. The column effluent was monitored at $215 \mathrm{~nm}$. Fractions were collected manually and used for mass spectrometric analyses as described in subsequent text.

\section{Synthetic Peptides}

The HPLC purified peptides were dissolved in water to yield a concentration of approximately 5-10 $\mathrm{pmol} / \mu \mathrm{L}$. The same sample amount, approximately $0.5 \mu \mathrm{L}$, was loaded for each experiment.

\section{Mass Spectrometry}

The primary molecular mass determination of the fractions and the synthetic peptides and the PSD experiment were performed on a VG TofSpec SE MALDI-time-of-flight (TOF) mass spectrometer (VG Analytical Ltd., Manchester, UK). One microliter of each HPLC fraction was mixed with $1 \mu \mathrm{L}$ of 4 -hydroxy- $\alpha$-cyano-cinnamic acid matrix or 2,5-dihydroxybenzoic acid (DHB) solution (Hewlett-Packard, Palo Alto, CA), and half of this mixture was loaded onto the sample cylinder.

One microliter of each HPLC fraction and $1 \mu \mathrm{L}$ of 4-hydroxy- $\alpha$-cyano-cinnamic acid matrix solution (saturated in a water/acetonitrile 70:30 mixture acidified with $0.1 \%$ TFA) were mixed and loaded for MALDI-CID analysis. These experiments were performed on a VG AutoSpec 5000 orthogonal acceleration (oa)-TOF tandem mass spectrometer. Figure 1 shows the ion optical design of this instrument. MS-1 is a VG AutoSpec 5000 double-focusing magnetic sector mass spectrometer equipped with a MALDI source. The $\mathrm{N}_{2}$ laser (337 $\mathrm{nm}$ wavelength) used in the MALDI source typically is operated at $10 \mathrm{~Hz}$ pulse rate. MS-1 is tuned to transmit just the ${ }^{12} \mathrm{C}$ monoisotopic ions of the precursor mass. Figure 2 shows an enlarged schematic of the gas collision cell and orthogonal acceleration-TOF housing. A two-stage deceleration electrostatic lens focuses the ions into an approximately parallel beam before they enter the gas collision cell $(l=5 \mathrm{~cm})$. Using xenon as the collision gas and a collision energy of $800 \mathrm{eV}$, the pressure in the cell was adjusted such that the selected precursor species was reduced to approximately $30 \%$ of its value without gas in the collision cell. Previous measurements on renin

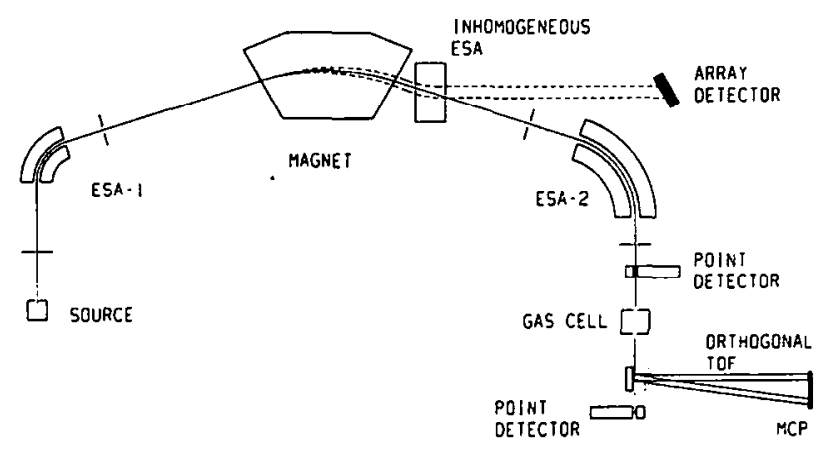

VG Au toSpec-FPD-TOF

Figure 1. Ion optical configuration of the VG AutoSpec-oa-TOF. 


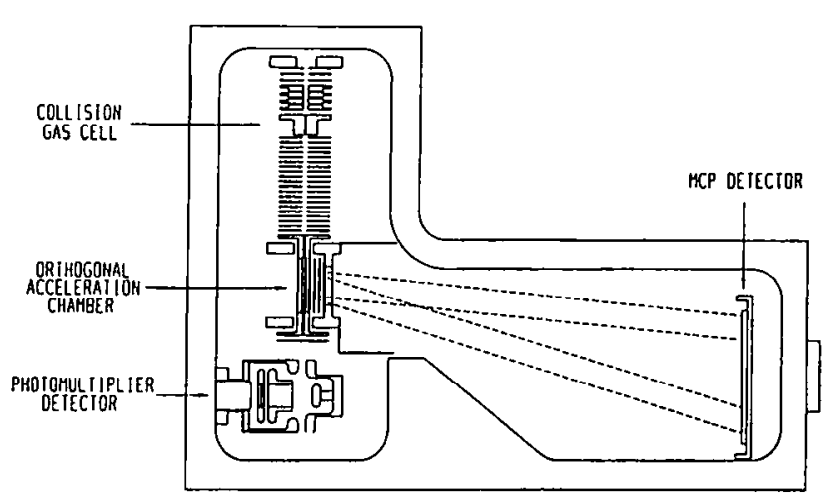

Figure 2. Schematic of the gas collision cell and the orthogonal acceleration-TOF.

substrate that used LSIMS established that attenuation of the selected ion by $30 \%$ corresponded to the ion gauge reading given in the following text. The calculated probability at $30 \%$ attenuation is $36 \%$ one- and $22 \%$ two-ion xenon atom collisions for precursor ions that transit the gas cell $[16,17]$. Under these circumstances the indicated average pressure on the ion gauge is $1.2 \times 10^{-6}$ mbar, which corresponds to a true pressure for xenon of approximately 4.0-5.5 $\times 10^{-7}$ mbar. The resulting mixture of precursor and product ions passes through the orthogonal acceleration chamber, which is normally field-free, but in which an electric field orthogonal to the direction of travel is established periodically by the sudden application of a voltage to the "push-out" electrode. The required precision for the timing of this extraction field is not excessive; for example, for precursor ions of mass $1000 \mathrm{u}$ and a collision energy of $800 \mathrm{eV}$ the required delay time is 75 $\mu \mathrm{s}$, with a transit window of $2 \mu \mathrm{s}$. This delay time is automatically calculated and controlled by the data system. The ions in the extraction field at this time are accelerated to ground potential through a two-stage voltage gradient into a linear TOF mass analyzer. The linear TOF has an effective path length of approximately $0.48 \mathrm{~m}$. The extraction pulse also denotes the start of the time-of-flight measurement. When these ions have left the orthogonal acceleration chamber, the push-out voltage is reset and ready for the next laser pulse. For this mode of operation the sampling efficiency is $100 \%$, which leaves no residual signal to be observed on detector 2. The TOF detector consists of $3 \times 50 \mathrm{~mm}$ long multichannel (MCP) plates (total length of $150 \mathrm{~mm}$ ). MS-2 always automatically records the full product ion spectrum irrespective of the precursor ion mass selected by MS-1. The ion signal from the microchannel plate detector is acquired by using a time-to-digital convertor (TDC) supplied by Precision Instruments (Knoxville, TN). The TDC acquisition rate is $1 \mathrm{GHz}$ and data are summed in real time into a $32 \mathrm{~K}$ word length histogram, which thereby allows acquisitions up to $32.7 \mu \mathrm{s}$. For a source voltage of $8 \mathrm{kV}$ and a collision energy of $800 \mathrm{eV}$, the mass range accommodated within the $32.7-\mu$ s flight time is $7200 \mathrm{u}$.
Time-of-flight calibration. All the fragment ions from the gas cell have essentially the same forward velocity component $\left(v_{x}\right)$. They all are accelerated exactly orthogonally with the same voltage $\left(V_{o}\right)$ to have the same energy $\left(e V_{o}\right)$, and hence all fragment ions have an orthogonal velocity component $\left(v_{y}\right)$ proportional to $\sqrt{ }\left(e V_{\mathrm{n}} / m\right)$. This means that all fragment ions have an orthogonal velocity component that is inversely proportional to the square root of the mass-as with normal time-of-flight analyzers - and all have the same orthogonal distance to travel to the plane of the detector. The fact that the ions also continue to drift forward as they travel to the detector is of no consequence to their time-of-flight, only to where they hit the detector. (Hence the need for a long detector.) Consequently, there is no need to apply any sort of correction to the normal time-of-flight calibration table. We have found that a two-point calibration works well, although it is our normal practice to generate a multipoint calibration from the product ion spectrum of $\mathrm{m} / \mathrm{z} 3510$ of cesium iodide.

The LSIMS-CID spectra were acquired on a Kratos Analytical (Manchester, UK) Concept tandem mass spectrometer of EBEB geometry, equipped with a continuous flow frit probe and a rapid scanning charge coupled device (CCD) detector ( $3 \mathrm{~s} /$ decade) $[8,18]$. A mixture of $5 \%$ thioglycerol, $5 \%$ acetonitrile, and $0.1 \%$ TFA in water was used as the liquid matrix at a flow rate of $3 \mu \mathrm{L} / \mathrm{min}$.

\section{Results}

\section{Synthetic and Standard Peptides}

A synthetic major histocompatability (MHC) peptide (APRTLVLLL, $\mathrm{MH}^{+}$at $m / z$ 995.6) was selected for evaluation of the ion types observed in MALDI-CID as well as for comparison with the well established LSIMS-CID and the newly emerging MALDI-PSD technique. These spectra are shown in Figure $3 a, b$, and c, respectively. All spectra exhibit $\mathrm{N}$-terminal fragment ions as expected because of the preferential charge retention at the Arg residue, as well as internal fragments. Each spectrum provides information on the amino acid composition in the form of immonium ions, but only the LSIMS-CID spectrum (panel b) shows abundant fragments due to side-chain losses from the pseudomolecular ion. The MALDI-CID spectrum (panel a) shows a complete $\mathbf{d}$ ion series at $\mathrm{m} / \mathrm{z} 382$ and $384\left(d_{4}, T h r\right.$ residues may yield two $d$ fragments), $469\left(d_{5}\right), 596\left(d_{6}\right), 681\left(d_{7}\right), 794\left(d_{8}\right)$, and $908\left(d_{9}\right)$. This spectrum also features abundant ions due to sequential neutral losses. These latter ions are similar to those observed in the PSD spectrum (panel c) described in subsequent text. For example, fragments PRT-17-18 at $m / z \quad 320, \mathbf{b}_{4}-17-18$ at $m / z \quad 391$, and $\mathbf{b}_{5}-17-18$ at $m / z$ 504 belong to this ion-type series. In the LSIMS-CID spectrum (panel b) the dominating fragments are a ions and the corresponding side-chain fragment $d$ ions. 


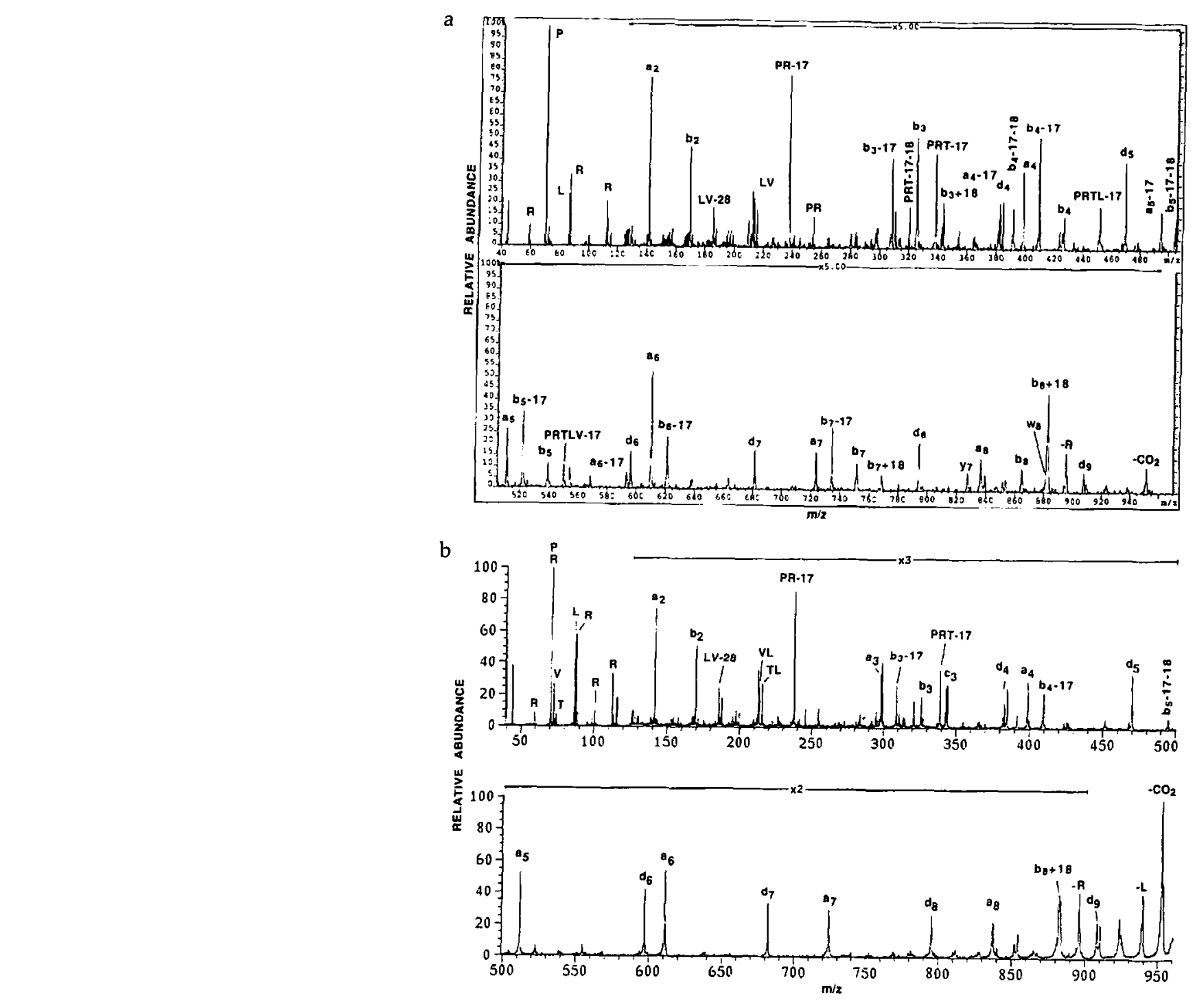

Figure 3. Fragmentation pattern of synthetic MHC peptide APRTLVLLL $\left(\mathrm{MH}^{+}\right.$at $\mathrm{m} / \mathrm{z}$ 995.6) by (a) MALDI-CID, (b) LSIMS-CID, and (c) MALDI-PSD.

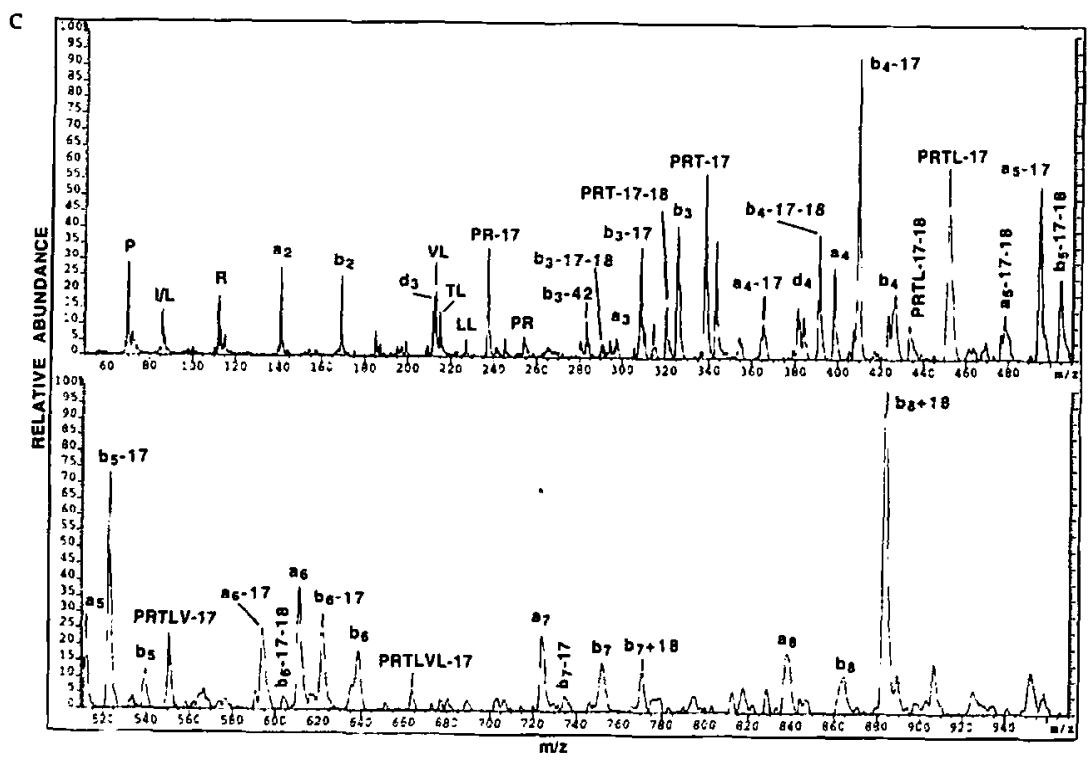



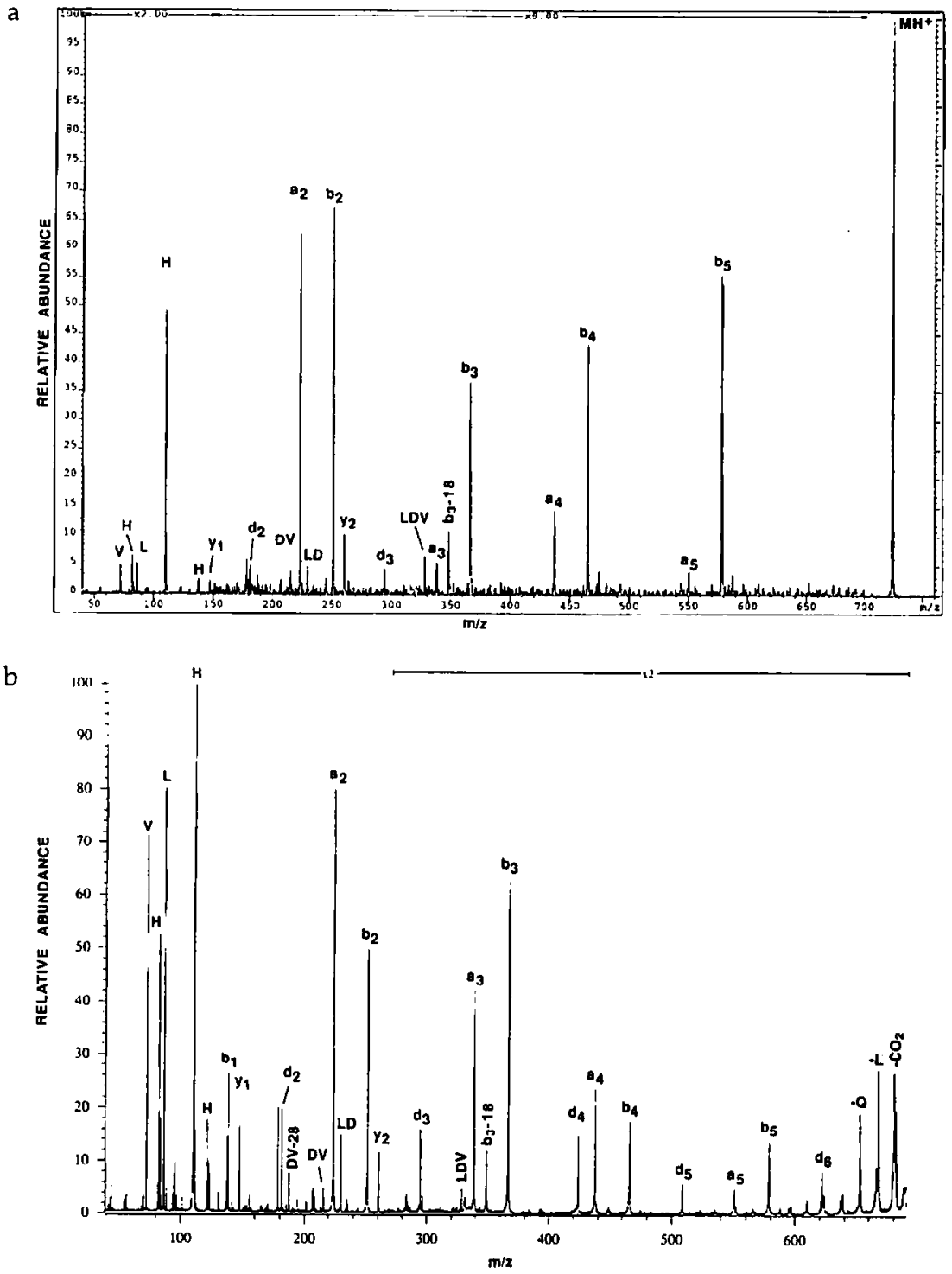

Figure 4. (a) MALDI-CID and (b) LSIMSCID of synthetic peptide HLDVLQ $\left(\mathrm{MH}^{+}\right.$ at $m / z$ 724.4).
This spectrum also exhibits a few ions formed via neutral losses, mostly $\mathrm{NH}_{3}$ losses $(-17 \mathrm{u})$. In the PSD spectrum (panel $c$ ) abundant ions are present due to sequential neutral losses from $\mathbf{a}$ and $\mathbf{b}$ ions as well as from internal fragments. Although this spectrum exhibited ions at masses that correspond to putative ions $d_{3}$ and $d_{4}$, it remains to be established whether this is in fact the case or whether they represent neutral loss or other unknown processes, because PSD spectra are not known to feature side-chain fragment ions.

The MALDI-CID and LSIMS-CID spectra of synthetic peptide (HLDVLQ, $\mathrm{MH}^{+}$at $m / z$ 724.4) are presented in Figure $4 a$ and $b$, respectively. The His residue, similarly to Arg, is a basic amino acid that retains the proton and thus controls the fragmentation. As expected, this peptide yields mostly $\mathrm{N}$-terminal ions. The MALDI-CID spectrum (panel a) gives immonium ions but lacks the fragments due to side-chain losses from the pseudomolecular ion. In this spectrum only $d_{2}$ and $d_{3}$ were detected from among the $d$ ions, and even their relative abundance is much lower than in the LSIMS-CID spectrum (panel b). The LSIMS-CID spectrum (panel b) exhibits abundant immonium ions and fragments due to the Leu and Gln side-chain cleavages from the pseudomolecular ion. The spectrum shows complete $\mathbf{a}$ and $\mathbf{b}$ ion series and the corresponding satellite $\mathbf{d}$ ions at $m / z 181\left(\mathbf{d}_{2}\right), 294$ $\left(d_{3}\right), 423\left(d_{4}\right), 508\left(d_{5}\right)$, and $621\left(d_{6}\right)$. Substituting Lys as the N-terminal amino acid of this peptide instead of His, only $d_{2}$ and $d_{4}$ were detected with similarly low abundance in the MALDI-CID analysis, whereas the LSIMS-CID spectrum exhibited a complete d series (data not shown).

MALDI-CID and LSIMS-CID spectra of another synthetic MHC peptide (LVMAPRTVL, $\mathrm{MH}^{+}$at $\mathrm{m} / \mathrm{z}$ 999.6) are shown in Figure 5a and b, respectively. Both spectra exhibit ions ( $v, w$, and $d$ fragments) that are usually observed only under high energy collision conditions. However, there are some obvious differences, not only in the relative intensities of the different ions, but in the presence or absence of certain types of fragments. While this is of little interpretative value, 


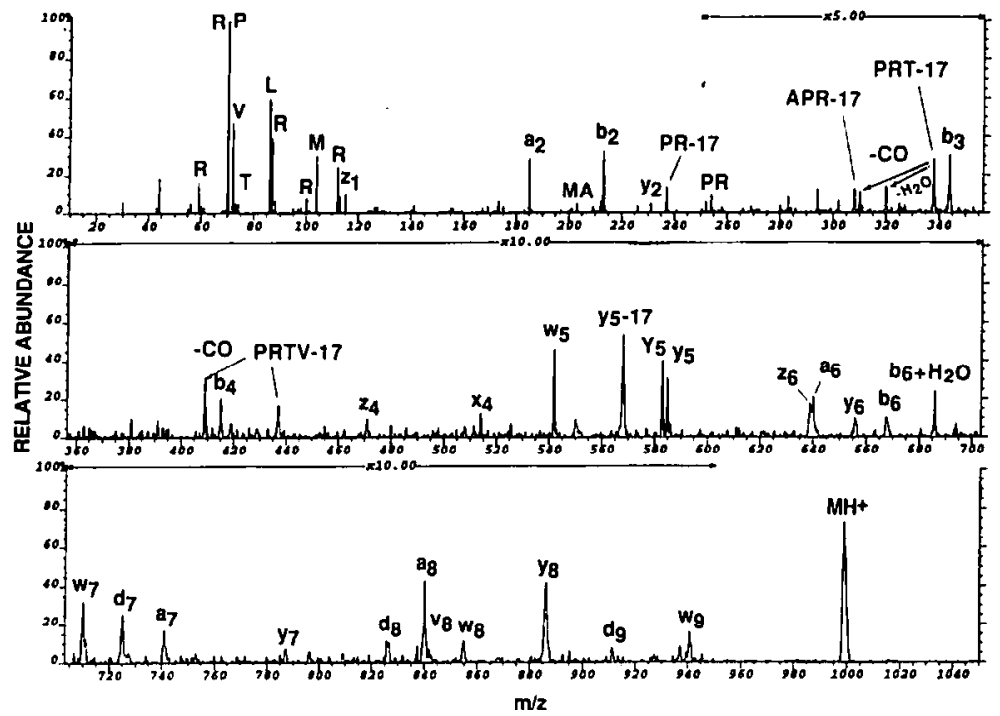

b
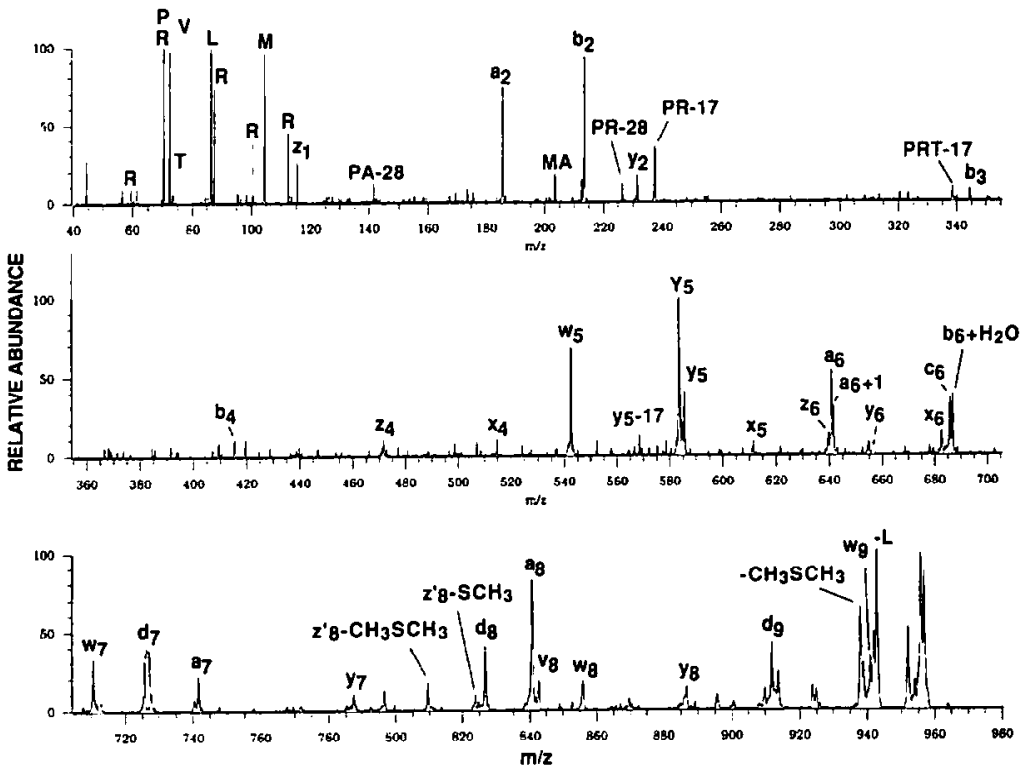

Figure 5. (a) MALDI-CID and (b) LSIMS-CID of synthetic MHC peptide LVMAPRTVL $\left(\mathrm{MH}^{+}\right.$at $m / z$ 999.6) once again the most striking difference is the lack of side-chain losses from the pseudomolecular ion in the MALDI-CID spectrum (panel a). The other interesting feature of this spectrum is a very abundant ion at $\mathrm{m} / \mathrm{z}$ 568 (i.e., $\mathbf{y}_{5}-17$ ). Ions at $m / z y_{i}-17$ usually are considered to be $\mathbf{z}_{i}$ ions. However, $\mathbf{z}$ ion formation requires a bond cleavage between the amino group and the $\alpha$-carbon of the amino acid. Because the fifth amino acid (starting from the C-terminus) is a Pro residue (an imino acid), this species cannot be a $z$ ion, but rather is the result of some other fragmentation processes. The loss of $17 \mathrm{u}$ (most likely $\mathrm{NH}_{3}$ ) from Arg-containing ions has been described previously, primarily in low energy CID spectra [19], but also has been reported in some high energy CID experiments (Figure 3b) [5].

To evaluate the quality of MALDI-CID spectra for a given number of laser shots from limited sample amounts, the CID spectrum of another synthetic MHC peptide (SPRYIFTML, $\mathrm{MH}^{+}$at $m / z$ 1127.6) obtained from 500 shots is shown in Figure 6. The immonium ions reveal the amino acid composition of the peptide [20]. From the sequence and satellite ions the order of the five N-terminal amino acids can be determined readily, and although the $d_{5}$ ion was not detected, a $\mathbf{d}_{6, \text { Ile }}$ at $m / z 708$ identifies the fifth amino acid as Ile [21]. The rest of the sequence can be deciphered mostly from the satellite and internal fragments. No $d_{9}$ ion was observed in this spectrum; thus the C-terminal amino acid could be either of the isobaric Leu-Ile pair.

As an additional indication of the sensitivity that may be achieved readily with this new technique, the CID spectra of two standard peptides that are commonly used in many laboratories have been recorded by loading 1 pmol of each. The MALDI-CID spectrum of substance $P$ by using 2,5-dihydroxybenzoic acid (DHB) as the solid matrix is shown in Figure 7. This spectrum shows some of the features described above: (1) lack of side-chain fragments from the pseudomolecular ion, except $-Q / K$; (2) neutral losses from $\mathbf{a}$ and $\mathbf{b}$ 


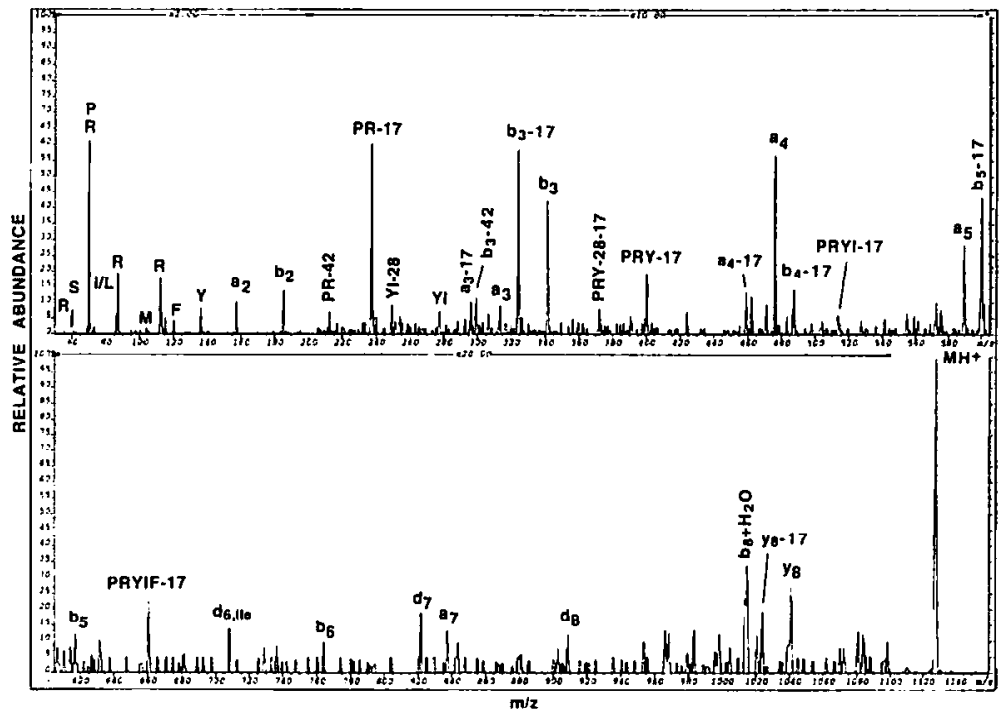

Figure 6. MALDI-CID spectrum of synthetic MHC peptide SPRYIFTML $\left(\mathrm{MH}^{+}\right.$at $m / z$ 1127.6), obtained from 500 laser shots at a single spot on the target loaded with $\sim 5$ pmol peptide. ions; (3) satellite ions, which are commonly observed in LSIMS high energy CID spectra from instruments of varying geometry (for comparison, see Table III in ref 22). Figure 8 shows the MALDI-CID spectrum of renin substrate. As is the case for its LSIMS high energy CID spectrum, this MALDI-CID spectrum exhibits a complete d ion series.

\section{Peptides from Unknown Protein}

The tryptic digest of an unknown soluble bacterial P450 protein was separated on a microbore C18 column. The fractions were collected manually and $1 \mu \mathrm{L}$ of each was analyzed by MALDI-TOF mass spectrometry, in reflectron mode, by using 4-hydroxy- $\alpha$-cyanocinnamic acid as the solid matrix. These unknown tryptic peptides were subjected to MALDI-CID analysis. The CID spectra of three components will serve to illustrate their interpretation. The pseudomolecular ions are at $m / z 781.4$ and 997.6 from fraction 12 and at $m / z 876.5$ from fraction 16 (chromatogram not shown). Figure 9 shows the MALDI-CID spectrum of the peptide with $\mathrm{MH}^{+}$at $m / z$ 781.4. The ions in the low mass region are significantly more abundant than those at higher masses. Although the amino acid composition can be determined readily from the immonium ions present- $m / z$ 59, 70, 87, 100, and 112 for Arg; $m / z 60$ for Ser; $m / z 72$ for Val; $m / z 120$ for Phe; $m / z$ 130, 159,170 for $\operatorname{Trp}[20]$ - no side-chain losses from the molecular ion were detected. The spectrum shows abundant ions with charge retention on both the $C$ and the $\mathrm{N}$-termini. The sequence was determined as follows [5]: First the presence of the C-terminal Arg was established from the immonium ions and $y_{1}$ at $m / z 175$ consistent with the specificity of trypsin. The presence of a $\mathbf{v}_{2}$ ion at $\mathrm{m} / \mathrm{z} 230$ without the accompanying $\mathbf{w}_{2}$ fragment indicated an aromatic amino acid $\mathrm{N}$-terminal to the Arg. Additional fragment ions at $m / z 344\left(\mathbf{z}_{2}\right)$ and $361\left(\mathbf{y}_{2}\right)$ require this amino acid to be Trp. Similarly, ions at $m / z 416\left(\mathbf{v}_{3}\right), 491\left(\mathbf{z}_{3}\right)$, and 508 $\left(y_{3}\right)$ revealed the next amino acid to be Phe. The order of the remaining three $\mathrm{N}$-terminal amino acids was determined from the $\mathrm{N}$-terminal fragment ions observed at $m / z 147\left(\mathbf{a}_{2}\right), 256\left(\mathbf{a}_{3}\right)$, and $274\left(\mathbf{b}_{3}\right)$, and supported by weak $C$-terminal fragments at $\mathrm{m} / \mathrm{z} 590$ $\left(z_{4}\right)$ and $678\left(z_{5}+1\right)$. The ion at $m / z 421$ is fragment $\mathbf{b}_{4}$, whereas that at $m / z 625$ corresponds to $\mathbf{b}_{5}+\mathrm{H}_{2} \mathrm{O}$. The sequence of this peptide is Ser-Ser-Val-Phe-TrpArg.

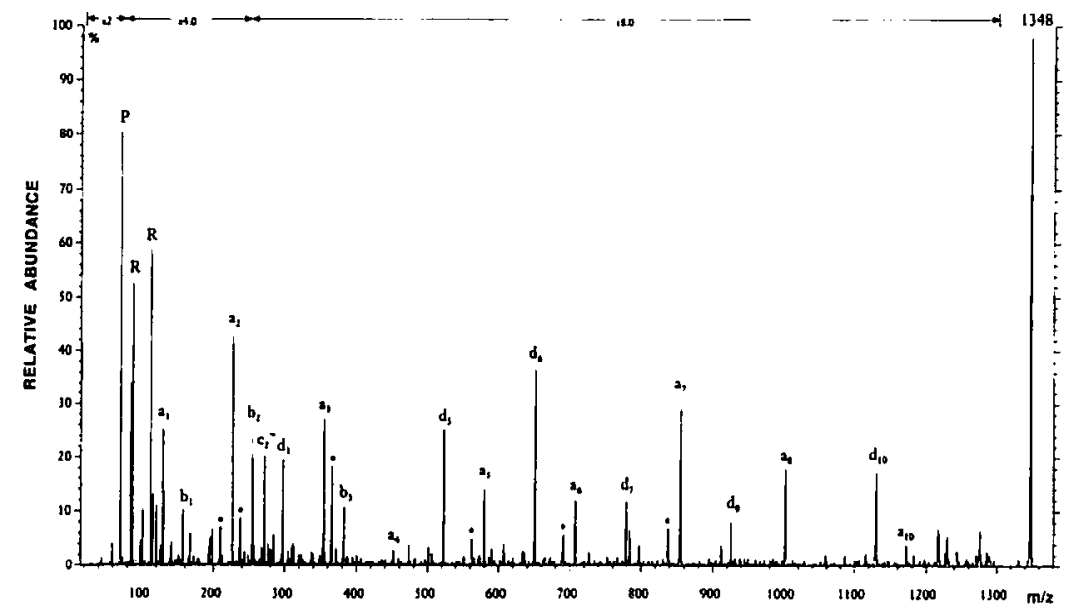

Figure 7. MALDI-CID spectrum of $1 \mathrm{pmol}$ (loaded) substance $P$ (RPKPQQFFGLM- $\mathrm{NH}_{2}$ ) with $\mathrm{DHB}$ as the solid matrix. Fragment ions labeled with asterisks are present due to neutral losses $(-17)$ from $a$ and $b$ sequence ions. Unusual satellite ions $a_{7}+1-Q$ and $d_{7, Q}$ were observed at $m / z 783$ and 797 , respectively. 
Figure 8. MALDI-CID spectrum of $1 \mathrm{pmol}$ (loaded) renin substrate (DRVYIHPFHLLVYS) with $\mathrm{DHB}$ as the solid matrix.

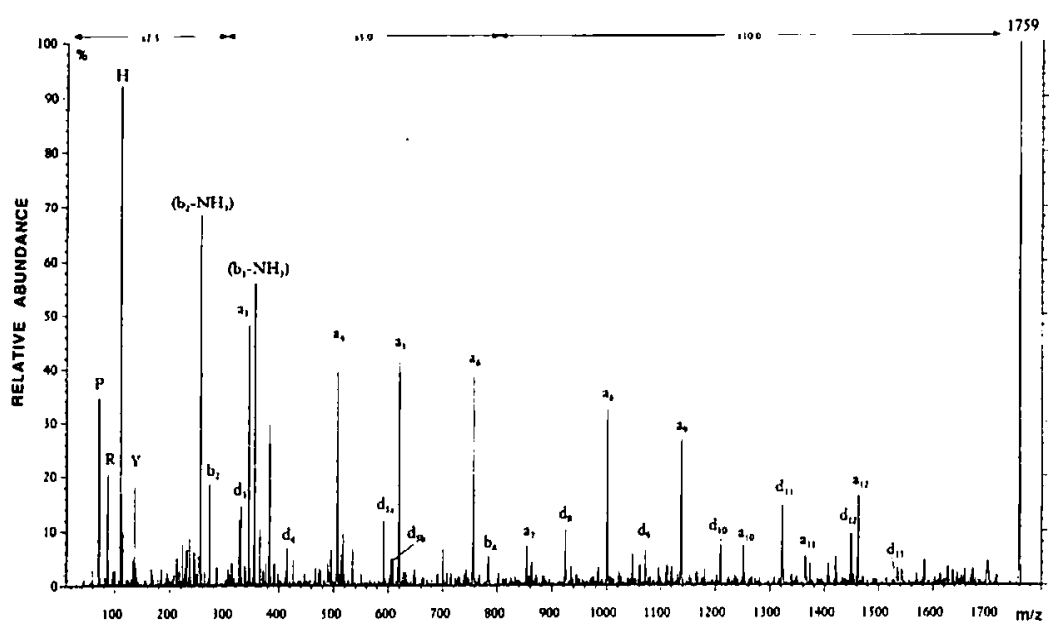

Figure 10 shows the MALDI-CID spectrum for the peptide with $\mathrm{MH}^{+}$at $m / z$ 997.6. The immonium ion region clearly indicates the presence of $\mathrm{Arg}$, Val, Met, Phe, and Tyr. Pro (immonium ion at $m / z 70$ ) and Asn (87) also could be present, but the presence of these amino acids may be masked by isobaric ions that arise from the Arg. The sequence was determined based upon the C-terminal satellite ion series, by drawing conclusions from the presence or absence of $\mathbf{v}$ and $\mathbf{w}$ ions [5]. For example, the absence of $\mathbf{v}_{2}$ or $\mathbf{w}_{2}$ ions suggested the presence of Gly or Ala in the penultimate position, whereas the $v_{3}$ (at $m / z$ 287) and $w_{3}$ (at $m / z$ 300) pair indicated the presence of a $\beta$-substituted amino acid, which must be Val, because the two other amino acids that bear a $\beta$-substituent (Thr and Ile) are not present in this particular peptide (deduced from the lack of the corresponding immonium ions). Side-chain fragments were detected for all the amino acids that would be expected by considering known fragmentation pathways [21], such as Met at $m / z 385\left(\mathbf{w}_{4}\right)$ and $470\left(\mathbf{z}_{5}+1-\mathrm{SCH}_{3}\right)$ [23], Pro at $m / z 587\left(\mathbf{w}_{6}\right)$, and Tyr at $m / z 742\left(\mathbf{v}_{8}\right)$. In addition to the $\mathrm{C}$-terminal ions, abundant $\mathrm{N}$-terminal sequence ions up to $b_{7}$ and internal fragments up to five residues long such as PAMVG (at $m / z$ 456) are present. The sequence of this peptide is Phe-Tyr-Gly-Pro-Ala-MetVal-Gly-Arg. Interestingly, the ion at $m / z 591$ represents a new type of fragmentation process for Met. The mass value corresponds to an a- $\mathrm{CH}_{3} \mathrm{SH}$ species. Similar species were observed in other Met-containing peptides and in LSIMS-CID experiments as well.

The MALDI-CID spectrum of the peptide with $\mathrm{MH}^{+}$ at $m / z 876.5$ is presented in Figure 11. As in the previous examples, the amino acid composition of the peptide was determined from the immonium ions noted: Arg, Ser, Pro, Ile-Leu, Phe, and Trp. Subtraction of the sum of these residue weights plus the elements of water (corresponding to the peptide termini) with the additional proton from the precursor ion mass value suggested that Ala is the missing residue. The sequence was established as previously described. Satellite fragments aided the sequencing effort at $m / z$ $229\left(w_{2}\right)$ and $230\left(v_{2}\right)$ for the Ser, $388\left(v_{4}\right), 401\left(w_{4}\right)$, and $415\left(w_{4}^{\prime}\right)$ for Ile, and $500\left(w_{5}\right)$ for Pro, and N-terminal (such as $b_{2}, b_{3}$ ) and internal fragments (for example, PI, PIA and PIAS) provided additional confirmation. The sequence of this peptide is Trp-Phe-ProIle-Ala-Ser-Arg.
Figure 9. MALDI-CID of a bacterial P450 tryptic peptide with $\mathrm{MH}^{+}$at $m / z$ 781.6. The amino acid sequence was determined from this spectrum to be Ser-Ser-Val-Phe-Trp-Arg.

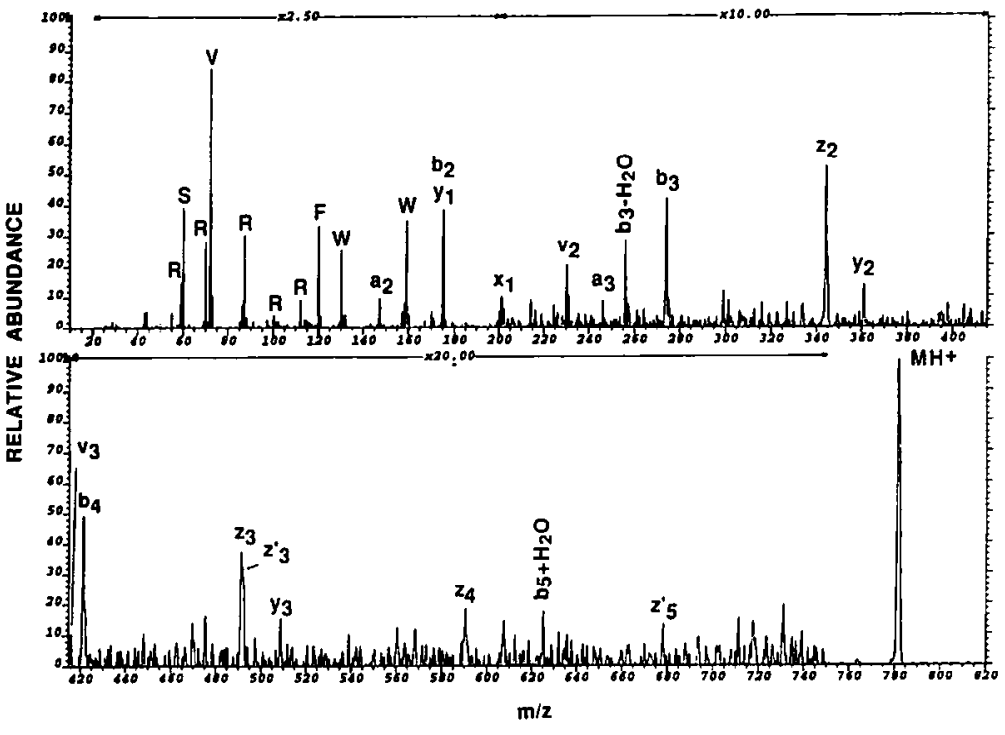




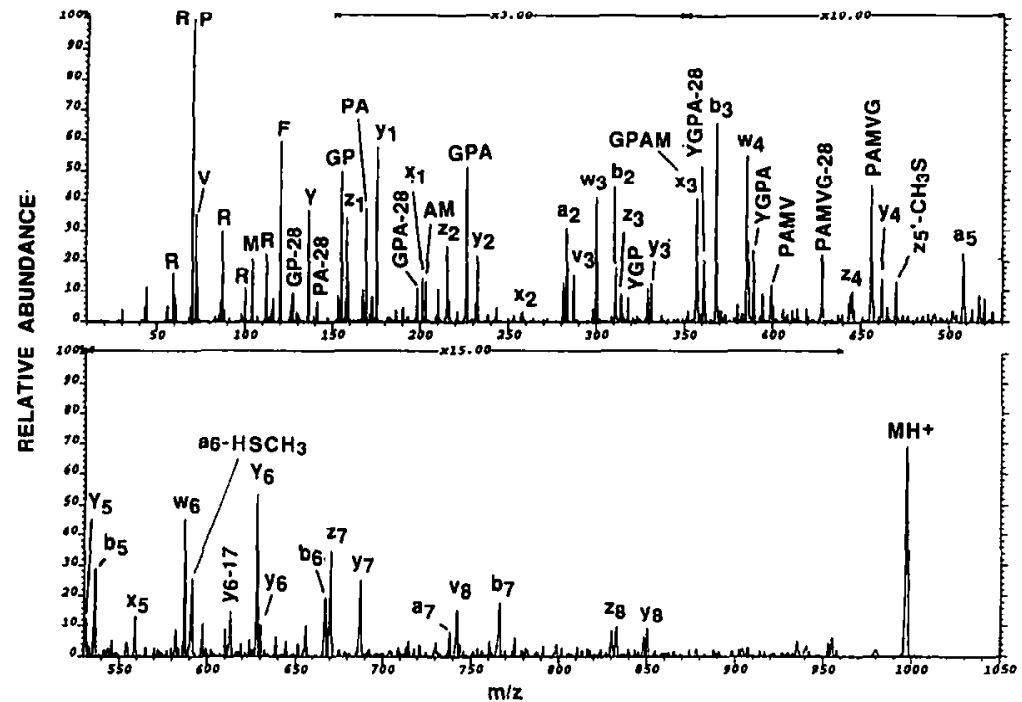

Figure 10. MALDI-CID of a bacterial P450 tryptic peptide with $\mathrm{MH}^{+}$at $m / z$ 997.6. The amino acid sequence was determined from this spectrum to be Phe-Tyr-Gly-Pro-Ala-Met-Val-Gly-Arg.

\section{Discussion and Conclusions}

In this study CID spectra of synthetic MHC and other peptides obtained by MALDI and LSIMS were compared qualitatively with one another (Figures 3-5) and with the corresponding MALDI-PSD spectrum (Figure 3). In addition, the power of a new tandem ion optical configuration (EBE-oa-TOF) designed to exploit the pulsed nature of the MALDI technique by using a $100 \%$ duty cycle to achieve optimal sensitivity is demonstrated by the sequence analysis of three peptides obtained from an unknown bacterial cytochrome P450 enzyme. Even in this initial work, sensitivity is demonstrated for the standard peptides substance $P$ and renin substrate with a loading of 1 pmol each to obtain the MAI.DI-CID spectra in Figures 7 and 8 . These MALDI-CID experiments were carried out by using a center-of-mass collision energy approximately a factor of 6 higher than that routinely employed in LSIMS-CID experiments by using a tandem instrument of EBEB geometry [22]. The MALDI-CID spectra ac- quired for these unknown peptides displayed fragmentation patterns directly comparable to those well established for four-sector high energy CID processes $[5,21]$. Abundant immonium and related ions revealed the amino acid composition of the peptides. Interestingly, although of little practical interpretative value, these same MALDI-CID spectra are devoid of sidechain losses from the pseudomolecular ion-abundant fragments commonly observed in LSIMS-CID spectra. The MALDI-CID spectra showed $\mathbf{v}, \mathbf{w}$, and/or $\mathbf{d}$ ions described earlier for high energy CID processes [21]. Concomitant with these ion series familiar from high energy CID spectra, additional ions are present that correspond to PSD-like fragmentation such as sequential neutral losses from arginine-containing fragment ions (Figure 3). In addition, unusually abundant $y-17$ ions were observed for $\mathrm{C}$-terminal ions formed by peptide bond cleavage at a Pro residue (Figures 5, 10, and 11). The virtually complete ion series with charge retention at the $\mathrm{N}$-terminus in the spectra depicted in Figures 9 and 10 are also somewhat unusual for tryptic

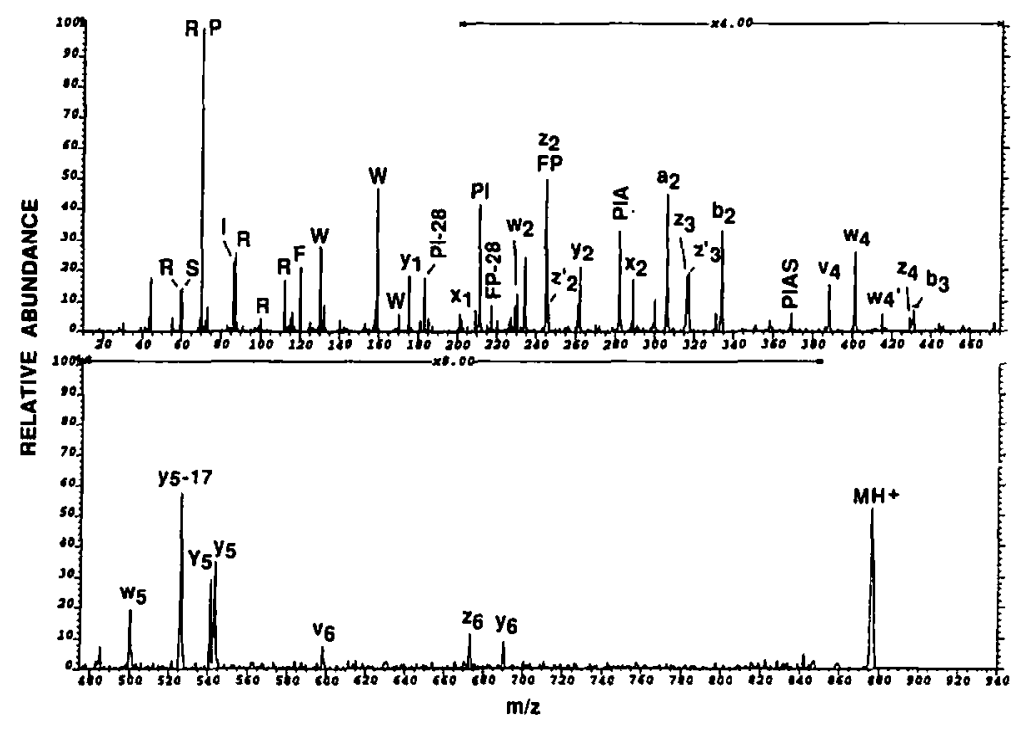

Figure 11. MALDI-CID of a bacterial P450 tryptic peptide with $\mathrm{MH}^{+}$at $m / z$ 876.5. The amino acid sequence was determined from this spectrum to be Trp-Phe-Pro-Ile-Ala-Ser-Arg. 
peptides, especially with an Arg residue at the $\mathrm{C}$ terminus. Similarly, the observation of $\mathrm{b}_{n-1}+\mathrm{H}_{2} \mathrm{O}$ has been reported in both low and high energy CID spectra only for peptides with preferential charge retention at the N-terminus [5, 24]. Although the CID spectra compared in Figures 3-5 were acquired on two tandem instruments of different geometry, we have evidence (based on LSIMS-CID spectra obtained from the same peptides on the two instruments compared; data not shown) that the unusual features of the MALDI-CID spectra are due to the different modes of ionization per se. Estimation of the sensitivity for MALDI-CID is presented in the data in Figures 6-8. The CID spectrum shown in Figure 6 was obtained from 500 laser shots taken at a single location on the sample probe tip that had been loaded with 5 pmol peptide, whereas the spectra of substance P (Figure 7) and renin substrate (Figure 8 ) were obtained by loading only 1 pmol of each peptide. Thus, it seems clear that femtomole level sensitivity will be achieved readily by incorporation of sample vials with limited volume [25].

The MALDI-CID spectrum of substance $P$ acquired in an earlier experiment on a four-sector mass spectrometer [13] did not show significant differences from that reported by LSIMS-CID [21]. However, in the present work certain fragment ions were observed via MALDI-CID (Figure 7), such as $\mathbf{b}_{1}, \mathbf{a}_{7}+1-Q$, and $\mathbf{d}_{7, Q}$, which were not detected in the MALDI-CID four-sector experiment [13]. It is of interest to note that photodissociation (PD) experiments also were performed on MALDI-generated ions with the same instrumentation [14]. In the case of renin substrate, the MALDI-PD spectrum is significantly different from the published LSIMS-CID spectrum [26]. Although the LSIMS-CID spectrum exhibits a complete $d$ series $\left(d_{7}\right.$ is missing because Pro residues cannot produce $d$ ions), only $d_{10}$ and $d_{11}$ were recorded in the MALDI-PD spectrum. However, the MALDI-CID spectrum in this work does in fact closely resemble that obtained by the LSIMS-CID technique (see Figure 8).

Further studies are underway to determine those factors that may influence the fragmentation observed in MALDI-CID experiments, which include the solid matrix selection, laser energy, and collision gas pressure.

\section{Acknowledgments}

The authors thank Dr. James DeVoss for providing the bacterial P450 enzyme for this study. We thank Dr. Frank R. Masiarz and Dr. Constance M. John for providing the synthetic peptides. This work was supported by grants from the NSF Biological Instru- mentation Program (DIR 8700766) and NIH NCRR Biomedical Research Technology Program (RR 01614 to ALB).

\section{References}

1. Burlingame, A. L.; Boyd, R. K.; Gaskell, S. J. Anal. Chem. 1994, 66, 634R.

2. Burlingame, A. L.; Carr, S. A., eds. Mass Spectrometry in the Biological Sciences; Humana Press: Clifton, N], 1996.

3. Bean, M. F.; Carr, S. A.; Thorne, G. C.; Reilly, M. H.; Gaskell, S. J. Anal. Chem. 1991, 63, 1473.

4. Biemann, K. Methods Enzymol. 1990, 193, 455-479.

5. Medzihradszky, K. F.; Burlingame, $\Lambda$. L. Methods: A Companion to Methods in Enzymology 1994, 6, 284.

6. Beavis, R. C.; Chait, B. T. Proc. Natl. Acad. Sci. USA 1990, 84, 6873.

7. Henzel, W. J.; Grimley, C.; Bourell, J. H.; Billeci, T. M.; Wong, S. C.; Stults, J. T. Methods: A Companion to Methods in Enzymology 1994, 6, 239-247.

8. Clauser, K. R.; Hall, S. C.; Smith, D. M.; Webb, J. W.; Andrews, L. E.; Tran, H. M.; Epstein, L. B.; Burlingame, A. L. Proc. Natl. Acad. Sci. USA 1995, 92, 5072.

9. Strobel, F. H.; Preston, L. M.; Washburn, K. S.; Russell, D. H. Anal. Chem. 1992, 64, 754.

10. Strobel, F. H.; Russell, D. H. Anal. Chem. 1992, 64, 2879.

11. Strobel, F. H.; Solouki, T.; White, M. A.; Russell, D. H. I. Am. Soc. Mass Spectrom. 1991, 2, 91.

12. Strobel, F. H.; Russell, D. H. Proceedings of the 38th ASMS Conference on Mass Spectrometry and Allied Topics; Tucson, AZ, 1990; p 1466.

13. Annan, R. S.; Köchling, H. J.; Biller, J. E.; Hill, J. A.; Biemann, K. Proceedings of the 40th ASMS Conference on Mass Spectrometry and Allied Topics; Washington, DC, 1992; pp 348-349.

14. Köchling, H. J.; Hill, J. A.; Biller, J. E.; Biemann, K. Proceedings of the 41st ASMS Conference on Mass Spectrometry and Allied Topics; San Francisco, CA, 1993; pp 435a-435b.

15. Bateman, R. H.; Green, M. R.; Scott, G. Procecdings of the 42 nd ASMS Conference on Mass Spectrometry and Allied Topics; Chicago, IL, 1994; p 1034.

16. Neumann, G. M.; Sheil, M. M.; Derrick, P. J. Z. Naturforsch. $1984,39 a, 584$.

17. Holmes, J. L. Org. Mass Spectrom. 1985, 20, 169.

18. Burlingame, A. L. In Biological Mass Spectrometry: Present and Future; Matsuo, T.; Caprioli, R. M.; Gross, M. L.; Seyama, Y., eds.; Wiley: New York, 1994; p 147.

19. Hunt, D. F.; Yates, J. R., III; Shabanowitz, J.; Winston, S.; Hauer, C. R. Proc. Natl. Acad. Sci. USA 1986, 83, 6233.

20. Falick, A. M.; Hines, W. M.; Medzihradszky, K. F.; Baldwin, M. A.; Gibson, B. W. J. Am. Soc. Mass Spectrom. 1993, 4, 882.

21. Johnson, R. S.; Martin, S. A.; Biemann, K. Int. I. Mass Spectrom. Ion Processes 1988, 86, 137.

22. Hayes, R. N.; Gross, M. L. Methods Enzymol. 1990, 193, 237

23. Downard, K. M.; Biemann, K. J. Mass Spectrom. 1995, 30, 25.

24. Thorne, G. C.; Gaskell, S. J. Rapid Commun. Mass Spectrom. $1989,3,217$

25. Jespersen, S.; Niessen, W. M. A.; Tjaden, U. R.; van der Greef, J.; Litborn, E.; Lindber, U.; Roeraade, J. In Mass Spectrometry in the Biological Sciences; Burlingame, A. L.; Carr, S. A., eds.; Humana Press: Clifton, NJ, 1996; pp 217-226.

26. Martin, S. A. Proceedings of the 37th ASMS Conference on Mass Spectrometry and Allied Topics; Miami, FL, 1989; pp 858-859. 